\title{
Failure to successfully open a chronic total coronary occlusion is associated with preserved global, but impaired regional myocardial function
}

\author{
Lynne K Williams ${ }^{5}$, Gideon A Paul ${ }^{4}$, Idan Roifman ${ }^{4}$, Mohammad I Zia ${ }^{4}$, Bradley H Strauss ${ }^{4}$, Andrew M Crean ${ }^{5}$, \\ Alexander W Leber ${ }^{4}$, Alexander J Dick ${ }^{6}$, Graham Wright ${ }^{3}$, Kim A Connelly ${ }^{1,2^{*}}$
}

From 16th Annual SCMR Scientific Sessions

San Francisco, CA, USA. 31 January - 3 February 2013

\section{Background}

Non-randomized studies have reported a prognostic advantage with percutaneous coronary intervention (PCI) in the treatment of chronic total occlusions (CTO). However failure to cross and successfully open a CTO confers a worse clinical outcome. Quantitative assessment of global left ventricular (LV) function in those with failure to open a CTO has not demonstrated deleterious alterations in cardiac function/remodeling. We hypothesized that more sensitive techniques that assess regional wall motion, such as strain imaging, may identify segments where cardiac function is adversely affected in those with failure to open the CTO.

\section{Methods}

30 patients referred for PCI to a single vessel de novo CTO underwent CMR examination before/after PCI. Left ventricular $(\mathrm{LV})$ function and transmural extent of infarction (TEI) were assessed using standard cine SSFP and late gadolinium enhanced T1-weighted imaging on a 1.5T MRI system. Regional cardiac function was assessed from cine SSFP images using Velocity Vector Imaging software (VVI, Siemens) on 20 patients. Data from each segment was summated and presented as global longitudinal, circumferential and radial strain/strain rate. All data was analyzed blind to the outcome of PCI. Data is presented as mean $+/$ - SD or percent change [delta $(\Delta)$ ] in final value from baseline.

\section{Results}

Successful CTO opening (TIMI 3 flow) occurred in 63\% of patients. Ejection fraction (EF) increased post PCI within the successful group $(\Delta=+12 \pm 20 \%)$, p < $0.0003)$. Within the failed group there was no change EF $(\mathrm{p}=0.09)$. In those with dysfunctional but viable segments $(25 \%)$ successful PCI increased EF $(p=0.04)$ in

Table 1 Global and Regional function in CTO cohort

\begin{tabular}{|c|c|c|c|c|c|c|c|}
\hline & \multicolumn{3}{|c|}{ Successful (S) CTO re-opening } & \multicolumn{3}{|c|}{ Failed (F) CTO re-opening } & \multirow{2}{*}{$\frac{S \text { VS } F}{P}$} \\
\hline & Pre & Post & $P$ & Pre & Post & $P$ & \\
\hline Ejection Fraction (\%) & $50.3 \pm 12.6$ & $54.3 \pm 10.7$ & 0.0003 & $57.6 \pm 8.1$ & $56.6 \pm 8$ & 0.093 & 0.0001 \\
\hline Infarct size (\%) & $14.5 \pm 13.5$ & $15.9 \pm 11.9$ & 0.39 & $11.1 \pm 8.1$ & $14.6 \pm 8.1$ & 0.09 & 0.74 \\
\hline Global longitudinal strain (\%) & $-11 \pm 4$ & $-12 \pm 3$ & 0.17 & $-16 \pm 3$ & $-11 \pm 4$ & 0.02 & 0.02 \\
\hline Global longitudinal strain rate $(\mathrm{m} / \mathrm{s})$ & $-0.63 \pm 0.3$ & $-0.69 \pm 0.2$ & 0.34 & $-0.99 \pm 0.3$ & $-0.70 \pm 0.3$ & 0.01 & 0.01 \\
\hline Global circumferential strain (\%) & $-25 \pm 8$ & $-26 \pm 6$ & 0.21 & $-29 \pm 3$ & $-29 \pm 4$ & 0.3 & 0.51 \\
\hline Radial strain (\%) & $23 \pm 8$ & $24 \pm 8$ & 0.4 & $28 \pm 8$ & $27 \pm 3$ & 0.7 & 0.31 \\
\hline
\end{tabular}

Data is mean +/- SD.

${ }^{1}$ Medicine/Cardiology, St Michael's Hospital, Toronto, ON, Canada

Full list of author information is available at the end of the article

(c) 2013 Williams et al; licensee BioMed Central Ltd. This is an Open Access article distributed under the terms of the Creative Commons Attribution License (http://creativecommons.org/licenses/by/2.0), which permits unrestricted use, distribution, and reproduction in any medium, provided the original work is properly cited. 


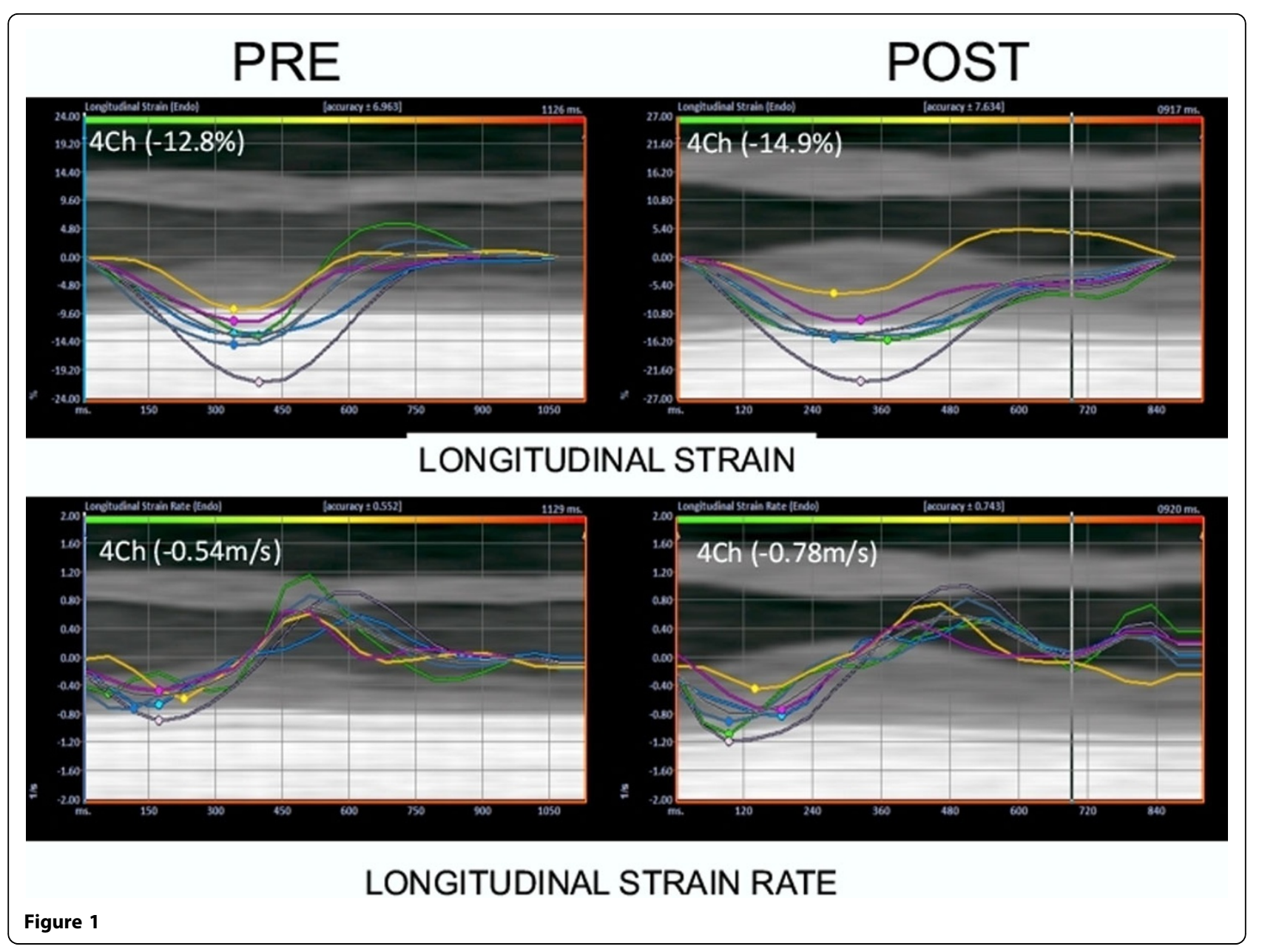

contrast to failed revascularization where the EF was not different.

Regional cardiac function varied significantly. Global longitudinal strain (GLS) fell significantly in the failed group $(\Delta=-25 \pm 17 \%, \mathrm{p}=0.02)$ in contrast to successful PCI where GLS did not change $(\Delta=+20 \pm 32 \%, \mathrm{p}=0.17)$. Global longitudinal strain rate followed a similar pattern to GLS (failed, $\Delta-30 \pm 17 \% \mathrm{p}<0.01$ versus success $\Delta+25$ $\pm 48 \% \mathrm{p}=0.34$ ). In contrast, radial and circumferential strain/strain rate were not different between groups after success/failed PCI (see table).

\section{Conclusions}

In this cohort, regional cardiac function assessment using Velocity vector imaging demonstrated a significant decline in GLS and GLSR in patients in whom PCI failed to open a CTO, with no change in global measures of cardiac function. Larger, randomized studies are required to assess the long-term benefits and morbidity of PCI in the treatment of CTOs, and potential impact of failure upon function and outcomes.

\section{Funding}

Funding was provided in part by the CIHR, Canada.

\section{Author details}

${ }^{1}$ Medicine/Cardiology, St Michael's Hospital, Toronto, ON, Canada. ${ }^{2}$ Medicine, Keenan Research Centre at the Li Ka Shing Knowledge Translation Institute, St Michael's Hospital, Toronto, ON, Canada. ${ }^{3}$ Medical Biophysics, University of Toronto, Toronto, ON, Canada. ${ }^{4}$ Schulich Heart Centre, University of Toronto, Toronto, ON, Canada. ${ }^{5}$ Department of Medical Imaging, Toronto Congenital Cardiac Center for Adults, Toronto, General Hospital, University of Toronto, Toronto, ON, Canada. ${ }^{6}$ Cardiology, Ottawa Heart Centre, Ottawa, ON, Canada.

Published: 30 January 2013

\section{doi:10.1186/1532-429X-15-S1-P224}

Cite this article as: Williams et al:: Failure to successfully open a chronic total coronary occlusion is associated with preserved global, but impaired regional myocardial function. Journal of Cardiovascular Magnetic Resonance 2013 15(Suppl 1):P224. 